\title{
Study of the Management of Diabetic Metabolic Emergency in the National Teaching Hospital HKM of Cotonou
}

\author{
Annelie Kerekou1, Eugène Zoumenou², Médard Agbantey², Corneille Tiomon², \\ Daniel Amoussou-Guenou', François Djrolo', Martin Chobli' \\ ${ }^{1}$ Clinical Service of Endocrinology and Metabolism, National Teaching Hospital HKM of Cotonou, University of \\ Abomey, Calavi, Benin \\ ${ }^{2}$ Clinical Service of Emergency Aid, National Teaching Hospital HKM of Cotonou, University of Abomey, Calavi, \\ Benin \\ Email: kerekouannelie@yahoo.fr
}

Received 11 September 2014; revised 8 October 2014; accepted 5 November 2014

Copyright (C) 2014 by authors and Scientific Research Publishing Inc.

This work is licensed under the Creative Commons Attribution International License (CC BY).

http://creativecommons.org/licenses/by/4.0/

(c) (i) Open Access

\section{Abstract}

Objective: To study the epidemiological, clinical and therapeutic profile of diabetic metabolic emergencies. Patients and methods: This was a prospective study in descriptive and analytical referred conducted over a period of 6 months in the National Teaching Hosptial HKM of Cotonou December 1, 2011 to May 31, 2012. The patients included in the case of our study were those who, conscious or comatose, had submitted a diabetic hypoglycemia or abnormal blood sugar (2.5 g/l), with a positive glycosuria and ketonuria positive or not. All patients gave their consent for this study. Results: 2786 patients were admitted to the emergency room, $57(2 \%)$ of acute metabolic decompensation of diabetes. DKA accounted for $1.1 \%$, hyperosmolar hyperglycemic syndrome $0.5 \%$ and $0.4 \%$ hypoglycemia. For hyperglycemic decompensation, sex ratio was 0.8 for females with a mean age of $50.7 \pm 16.9(16-84)$. For hypoglycemia, male gender was predominant. As decompensation factors for hyperglycemia, infection was found in $54 \%(n=30)$ of cases and stroke by $29 \%$ hypertension $(n=15)$. As for hypoglycemia triggers were dominated by dietary error $(50 \%)$ and therapeutic errors $(25 \%) .63 \%(n=36)$ of patients underwent resuscitation. More than 3 out of 4 patients were resuscitated to insulin. $98 \%$ of patients were rehydrated. The outcome was favorable in $56 \%$ of cases. The death rate was $25 \%(n=14)$. Conclusion: Diabetes mellitus is a serious condition and its severity is mainly due to complications which can be acute or chronic.

\section{Keywords}

Ketoacidosis, Diabetes, Decompensation, Ketosis 


\section{Introduction}

Diabetes mellitus is one of the most common in the world of non-communicable diseases (NCDs). It is the fourth or fifth leading cause of death in most high-income countries, and many data now tend to prove that reached epidemic proportions in many developing and newly industrialized countries. This is a serious condition and its severity is mainly due to complications which can be acute or chronic.

According to the Atlas of the IDF Diabetes, 5th Edition, “The estimated number of adults living with diabetes number exploded in 2011 to 366 million, or $8.3 \%$ of the global adult population this number is expected to reach 552 million people by 2030 (9.9\% of adults), which corresponds to the discovery of about three more cases of diabetes every 10 seconds” [1].

In Africa, the available data on NCDs are scarce, incomplete and fragmented [2]. However, we know that diabetes is strongly emerging and BENIN is certainly not immune. In 2008, the overall prevalence of diabetic hyperglycemia kind in Benin was 2.6\% [3], while in 2001 Djrolo et al. had obtained a prevalence of $1.1 \%$ from a study of 2362 subjects divided in 12 departments of the country [4].

In Benin, diabetes is part of the National Programme for the Fight against Non-Communicable Diseases (PNLMNT). In this respect at national level, it is organized regularly, for an early care, activities of routine screening by various agencies. Among these, we have the center screening, treatment and monitoring of Cotonou (Akpakpa Diabetes Center) created in 1995 by LION'S CLUB INTERNATIONAL, with financial assistance from the Commission of the European Communities and the Department of French Cooperation. Notwithstanding these provisions, he observed an increase in cases of complications of diabetes, due to unawareness of manifestations and severity of the disease and the low socio-economic level.

Indeed, the rate of occurrence of hospital ketotic decompensation was $21.4 \%$ in a diabetic population studied in Benin [5]. A general referral hospital in Kinshasa, in a diabetic population studied, 40.1\% had a diabetic coma and ketoacidosis 59.9\% [6]. According to E. Sobngwi, “75\% of diabetics are unaware of the diagnosis is fortuitous or placed in the context of a frank symptomatic decompensation. The off springing clinics emergency presentations are often extreme, causing high mortality” [7]. This state of affairs has to be exacerbated by the precarious support of our health system, especially at the stage of acute decompensation views urgency.

Having looked at all the foregoing and having the need to "codify and harmonize the management of these patients" [7] admitted for hospital care reference Benin, we proposed to make a "study of the management of diabetic metabolic emergency in the National Teaching Hospital HKM of Cotonou”.

\section{Frame and Method of Study}

This was a prospective, descriptive and analytical study of a period of six months from 1st December 2011 to 31st May 2012.

All diabetic patients, known and unknown, received in the National Teaching Hospital of Cotonou HKM were concerned. The diagnosis of diabetes was chosen following WHO biological criteria.

The patients included in our study were those who, conscious or comatose, had submitted a diabetic hypoglycemia or abnormal hyperglycemia ( $\geq 2.5 \mathrm{~g} / \mathrm{l})$ with positive glycosuria and ketonuria positive or not.

Hyperglycemia or hypoglycemia patients are justified by the context of trauma or other circumstances, are systematically excluded.

Epidemiological and clinical variables were age, sex, profession, health insurance, family history of diabetes, primary knowledge of diabetes, the duration of diabetes, previous observation of diabetic treatment: Evil or not followed by treatment, when treatment is modified irregularly, either temporarily or permanently discontinued without medical advice, type of diabetes, time reference, obesity, hypertension, smoking, alcoholism, polyuria syndrome, asthenia, weight loss, vomiting, fever, abdominal pain, coma, respiratory disorders, agitation, state of hydration.

Paraclinical variables were capillary glycerin, glycosuria, ketonuria, serum potassium, serum sodium, white blood cell count (NB), blood culture, GE/DP, urine culture, (Urinalysis), chest radiograph, electrocardiogram (ECG), blood gases (BG).

Evolutionary and therapeutic variables were insulin recovery, general resuscitative measures comatose, length of hospital stay, evolution, output processing, glycemic profile, implemented protocols and compliance, instead of under observation, time management, regularity of care and compliance with prescribed protocols.

Data collection was to collect data from patients who can express themselves or their companions, observation 
and from medical records.

Entry and statistical analysis were made by the Epi Info version 6.04 dfr April 2001.

The graphics were developed using Microsoft Excel 2007 software.

\section{Results}

\subsection{Epidemiological Characteristics of Decompensation}

\subsubsection{Prevalence}

During the period of data collection, 2786 patients were admitted to the National Teaching Hospital among whom, 57 cases of acute decompensation of diabetes mellitus, a $2 \%$ incidence of metabolic diabetics' emergencies in the National Teaching Hospital HKM of Cotonou.

The $2 \%(\mathrm{n}=57)$ of cases of diabetic metabolic emergency that constitutes our study population was divided into 31/57 (54\%) of diabetic ketoacidosis representing half of the cases, 14/57 (25\%) of hyperosmolar hyperglycemia syndrome and 12/57 (21\%) hypoglycemia.

The impact of each of these types of decompensation of diabetes in the hospital would be:

-DKA: 31/2786 (1.1\%);

-Syndrome hyperosmolar hyperglycemia: 14/2786 (0.5\%);

-Hypoglycemia: 12/2786 (0.4\%);

-No diagnosis of lactic acidosis had been laid.

\subsubsection{Distribution of Patients by Sex}

$>$ Hyperglycemic decompensation

Among the 45 hyperglycemic decompensation included in the study, 20 patients were male (44\%) and 25 female (56\%) or a sex ratio of 0.8 .

$>$ Hypoglycemic decompensation

For hypoglycemia, $75 \%(n=9 / 12)$ men against $25 \%(n=3 / 12)$ for females.

Table 1 shows distribution of patients by age.

Table 2 shows distribution of patients according to the type of diabetes.

Table 3 shows hypoglycemic diabetes decompensation.

\subsection{Specific Features of Decompensation}

\subsubsection{Types Decompensation \\ -DKA: 31/2786 (1.1\%); \\ -Syndrome of hyperosmolar hyperglycemia: 14/2786 (0.5\%); \\ -Hypoglycemia: 12/2786 (0.4\%).}

\subsubsection{Distribution of Patients According to Socio-Professional Category and Health Insurance} Non-employees represent $72 \%(n=41 / 57)$ of our study population. $21 \%$ or 12 of 57 patients only have health insurance.

\subsubsection{Knowledge of Diabetic Field}

Of our patients, $77.2 \%(n=44 / 57)$ know themselves diabetics.

Figure 1 shows Inter current diseases as factors of decompensation.

Figure 2 shows Infectious seats.

Among the cases of skin infection seats, diabetic feet come in first place with $67 \%(n=6 / 9)$, a case of erysipelas was observed.

Figure 3 shows hypoglycemic decompensation set off.

Figure 4 shows clinical characteristics of hyperglycemic decompensation.

Figure 5 shows clinical features of hypoglycemic decompensation.

Table 4 shows capillary glucose.

Table 5 shows search glycosuria, ketonuria and electrolytes.

The only patient who had a negative ketonuria at the entrance, was a young (18 years) and had received insulin before admission (glucose $=4.49 \mathrm{~g} / \mathrm{l}$ and glycosuria $=4+$ ). 


\subsubsection{Other Biological Analyses}

-Of the 37\% (21/37) of patients who received WBC, $33 \%$ had leukocytosis, 19 of 57 patients.

-The GE/DP was positive in two patients, so $3.5 \%(2 / 57)$.

-Other laboratory tests expected to know: blood gas, urinalysis, blood culture, have been made or were not available.

\subsection{Support}

\subsubsection{Timeout Patients}

The average waiting time for patients at admission for first aid was $47 \mathrm{mn}$, with extremes of 20 minutes at 2:45. Influencing Factors:

Table 1. Distribution of patients by age by type of decompensation.

\begin{tabular}{ccc}
\hline Types of Decompensation & Average Age (year) & Extremes (years) \\
\hline Ketoacidosis & $46,161 \pm 18,827$ & $16-84$ \\
Hyperosmolar & $56,214 \pm 8702$ & $42-68$ \\
Hypoglycemia & $56,000 \pm 16,586$ & $24-75$ \\
\hline
\end{tabular}

The average age of patients for all decompensation was $50.702 \pm 16.927$ years with extremes of 16 and 84 years $(p=-0.000)$. The average age of patients in the hyperglycemic decompensation was $49.289 \pm 16.918$ years, ranging from 16 to 84 years $(p=-0.000)$.

Table 2. Distribution of patients according to the type of hyperglycemic diabetes decompensation.

\begin{tabular}{cccccc}
\hline Types of & \multicolumn{5}{c}{ Type of Diabetes } \\
\hline Decompensation & $\mathrm{N}$ & 1 & $\mathrm{n}$ & 2 \\
& & $\%$ & 17 & 38 \\
Ketoacidosis & 14 & 31 & 10 & 22 \\
Hyperosmolar & 4 & 9 & 27 & 60 \\
Total & 18 & 40 & & \\
\hline
\end{tabular}

$22 \%$ (10/45) of type 2 diabetic hyperosmolar coma were against 9\% (4/45) of type 1; 38\% (17/45) of type 2 diabetes have ketoacidosis against 31\% $(14 / 45)$ of type 1.

Table 3. Distribution of patients according to the type of diabetes for hypoglycemic decompensation.

\begin{tabular}{|c|c|c|c|c|}
\hline Types of & \multicolumn{4}{|c|}{ Type of Diabetes } \\
\hline \multirow[t]{2}{*}{ Decompensation } & \multicolumn{2}{|c|}{1} & \multicolumn{2}{|c|}{2} \\
\hline & $\mathrm{N}$ & $\%$ & $\mathrm{n}$ & $\%$ \\
\hline Hypoglycemia & 4 & 33 & 8 & 67 \\
\hline
\end{tabular}

$67 \%$ (8/12) of type 2 diabetes have hypoglycemia against 33\% (4/12) of type 1 diabetes.

Table 4. Distribution of patients according to the type of diabetes for hypoglycemic decompensation.

\begin{tabular}{ccc}
\hline Type of Decompensation & Glyc Average (g/l) & Extremes (g/l) \\
\hline All decompensation & $3.635 \pm 2.023$ & $0.20-6.07$ \\
Hyperglycemic & $4.497 \pm 1.263$ & $2.340-6.07$ \\
Acidocetosic & $4.075 \pm 1.152$ & $2.340-6.0$ \\
Hypoglycemic & $0.404 \pm 0.178$ & $0.2-0.64$ \\
\hline
\end{tabular}

-Among the hyperglycemic decompensation, 27\% ( $n=12 / 45)$ had a massive glucose $6 \mathrm{~g} / \mathrm{l}$

-Among the cases of ketoacidosis rather note $16 \%(n=5 / 31)$ with glucose $6 \mathrm{~g} / \mathrm{l}$.

-For hypoglycemia, $25 \%(n=3 / 12)$ have a severe low blood glucose $0.2 \mathrm{~g} / \mathrm{l}$. 
Table 5. Results of the search glycosuria, ketonuria and Ketoacidosis electrolytes for decompensation.

\begin{tabular}{|c|c|c|}
\hline & $\mathrm{n}$ & $\%$ \\
\hline \multicolumn{3}{|l|}{ Glycosuria } \\
\hline \multicolumn{3}{|l|}{ Negative } \\
\hline+ & 4 & 12.9 \\
\hline++ & 7 & 22.6 \\
\hline+++ & 14 & 45.2 \\
\hline++++ & 6 & 19.4 \\
\hline \multicolumn{3}{|l|}{ Ketonuria } \\
\hline Negative & 1 & 3.25 \\
\hline+ & 10 & 32.3 \\
\hline++ & 4 & 12.95 \\
\hline+++ & 8 & 25.8 \\
\hline++++ & 8 & 25.8 \\
\hline \multicolumn{3}{|l|}{ Potassium } \\
\hline Normal & 13 & 41.9 \\
\hline Low & 3 & 9.7 \\
\hline High & 1 & 3.2 \\
\hline Not done & 14 & 45.2 \\
\hline \multicolumn{3}{|l|}{ Natremia } \\
\hline Normal & 13 & 41.9 \\
\hline Low & 2 & 6.5 \\
\hline High & 2 & 6.5 \\
\hline Not done & 14 & 45.2 \\
\hline
\end{tabular}

-All patients had a positive urine glucose $\geq 65 \%++$

$-97 \%(30 / 31)$ of patients had a positive urine ketones $\geq 87 \%(27 / 31)++$.

Non-availability of specific emergency kits.

Mass-attendance of patients and lack of a sort of sick.

-The time of consultation, the night guard team is reduced compared with the influx of injured patients especially.

-Ruptures produced at the central pharmacy.

\subsubsection{Resuscitation}

(1) Measures general resuscitation cases of coma

$92 \%(n=33 / 36)$ of patients in coma had received oxygen $(3-6 \mathrm{~L} / \mathrm{min}), 42 \%(n=15 / 36)$ required orotracheal intubation.

The probabilistic antibiotic therapy was early in the amoxicillin and acid clavulanic or ceftriaxone. She was then rehabilitated according to the results of the balance sheet and changes.

(2) Electrolyte recovery

$98.2 \%$ of the patients underwent rehydration, 44 out of 45 .

As for electrolytes, there was an intake of potassium chloride in the second hour and sodium chloride in $86.1 \%$ of patients, 39 out of 45 . 


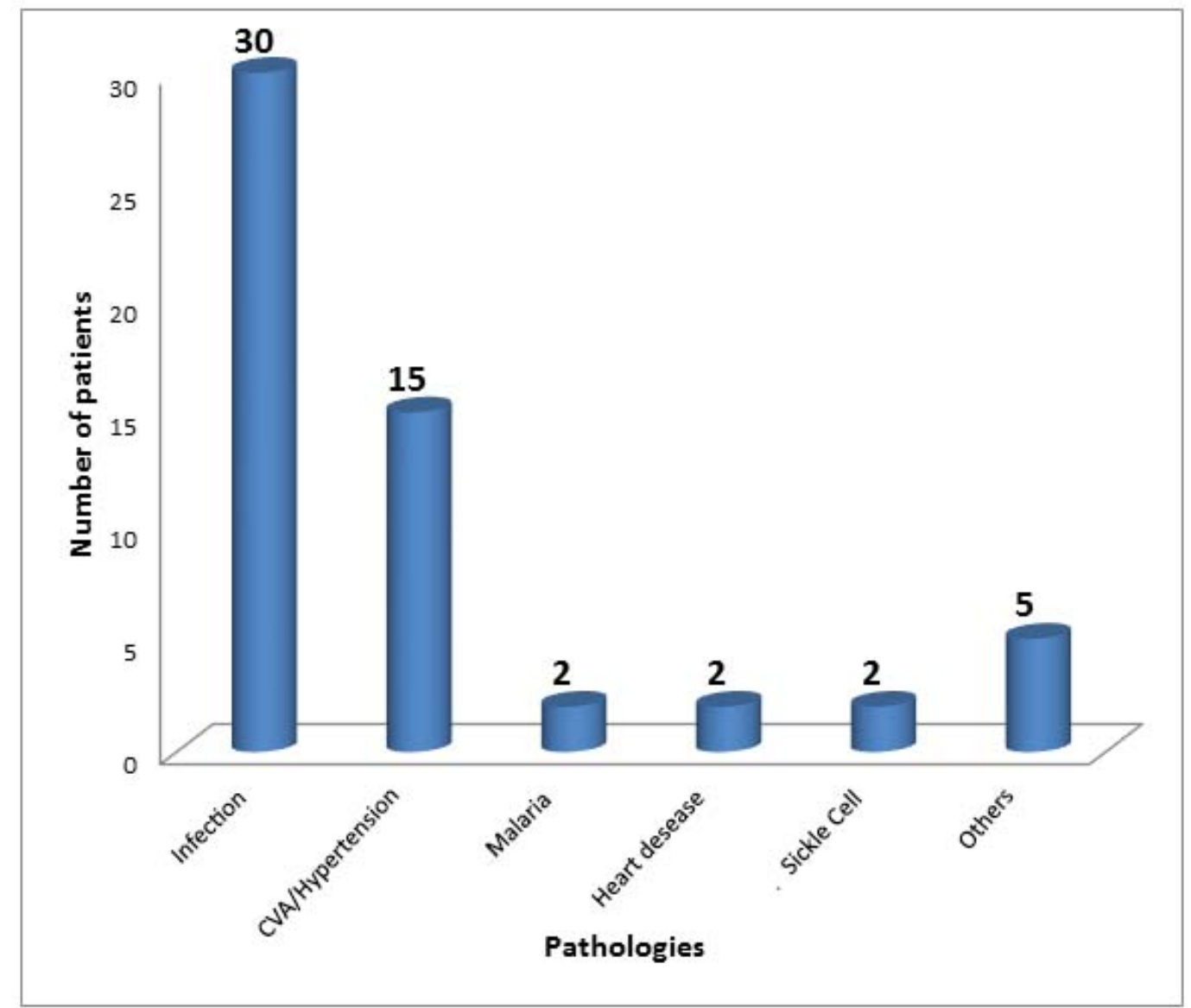

The group designated as "other" pathologies is defined as: trauma, liver cirrhosis, kidney disease, anemia and epistaxis each of which represents $1.8 \%(1 / 57)$.

Figure 1. Pathologies associated with decompensation.

(3) Intensive insulin

$75 \%$ of our patients underwent resuscitation insulin or 34 to 45 .

Discontinuous/h: 73\% (33/45).

Electric syringe pump 9\% (4/45).

4 times: $18 \%(8 / 45)$.

\subsection{Evolution}

3.4.1. Length of Stay of Patients in the Emergency Department

The average length of stay in the service is 4 days with extremes of 0 days to 25 days.

More than half of the study population, $63 \%(n=36 / 57)$ stayed less than 72 hours in service.

\subsubsection{Destination Patients}

Evolution favorable: 56\% (32/57).

Transfer in intensive care: 19\% (11/57).

Death: 25 \% (14/57).

\section{Discussion}

\subsection{On Epidemiological}

In our study, the prevalence of diabetic metabolic emergency was $2 \%$. 


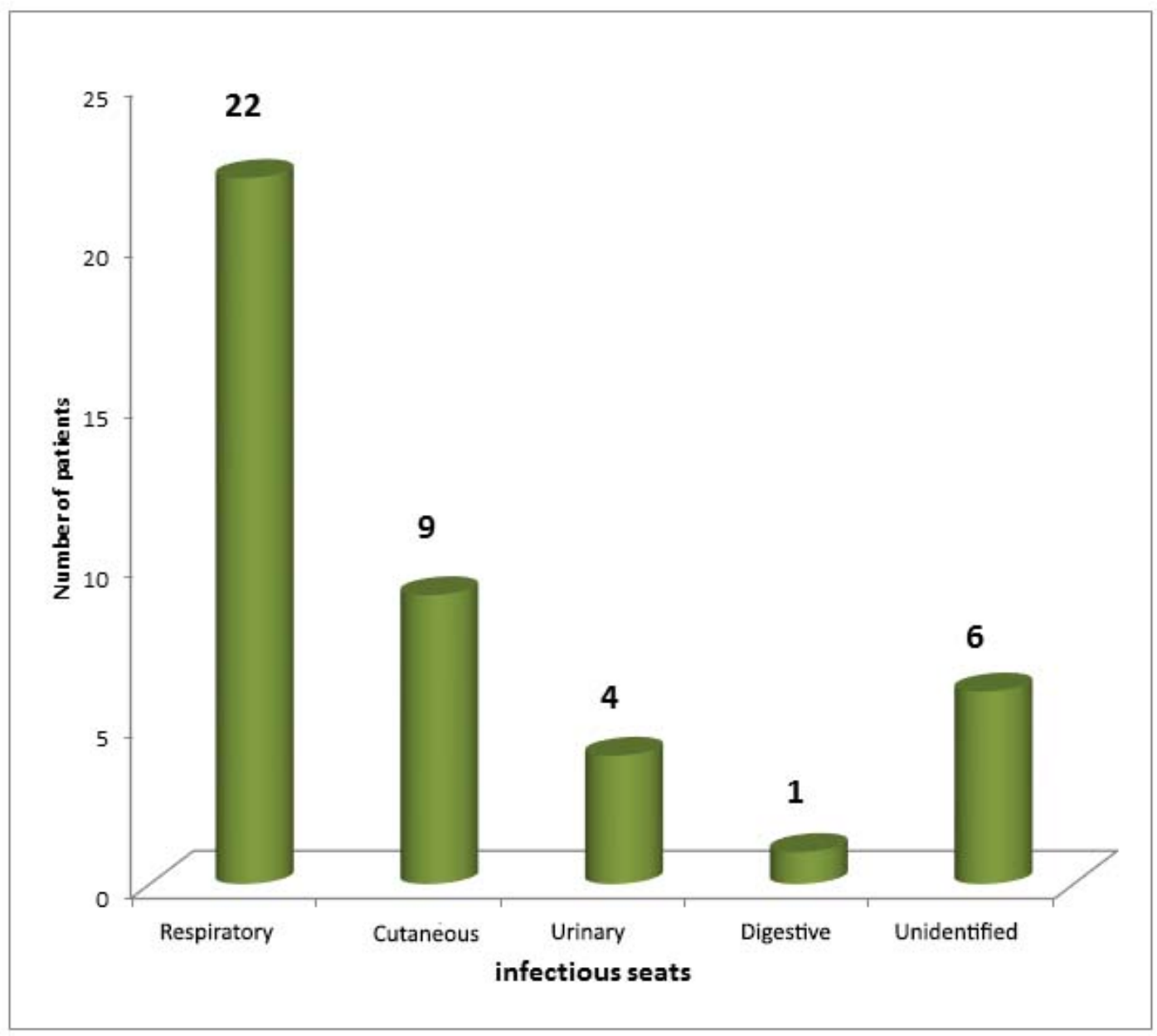

49\% (22/45) of patients who had a hyperglycemic decompensation had a respiratory infection.

Figure 2. Distribution of patients according to disease outbreaks.

The hyperglycemic decompensation types come in first place with $1.6 \%$ (1.1\% for diabetic ketoacidosis and $0.5 \%$ for hyperosmolarity) and hypoglycemic decompensation $0.4 \%$.

Kitabchi, A. E., et al. found in 2006 that ketoacidosis decompensation represent about $4 \%-9 \%$ of cases of hospitalization and hyperosmolarity of diabetics $1 \%$ [8].

These low rates obtained in our series can be explained by the diversity of pathologies admitted in the emergency department. Diabetics are rather drowned in the flood of other emergencies (81 diabetic patients in 2786) despite the specificity required for their support.

\subsubsection{Sex and Age}

For all types of acute metabolic decompensation of diabetes, the average age of patients in our study population was $50.702 \pm 16.927$ years with extremes of 16 and 84 years $(p=-0.000)$.

Regarding gender, the average age of the study population was $53.345 \pm 15.814$ years with extremes of 16 to 84 for males and $47.964 \pm 17.879$ years with extremes of 18 and 75 years for females and a sex ratio of 1.03.

In Madagascar in 2006, I. V. Ramanitriniaina et al. found similar results[9].

For acidocetosic decompensation, mean age was $46.161 \pm 18.827$ with a range of 16 - 84 years, which approximates the results obtained by Wade K. A. et al. in Senegal [10].

Among the 45 hyperglycemic decompensation included in the study, $44 \%(n=20)$ of patients were male and $56 \%(n=25)$ female, giving a sex ratio of 0.8 in favor of female the average age of patients being $49.289 \pm$ 16.918 years with a range of $16-84$ years.

In a similar study with $96.8 \%$ of hyperglycemic decompensation in 2002, Sidibe, A. T., et al. found $51.21 \pm 13$ 


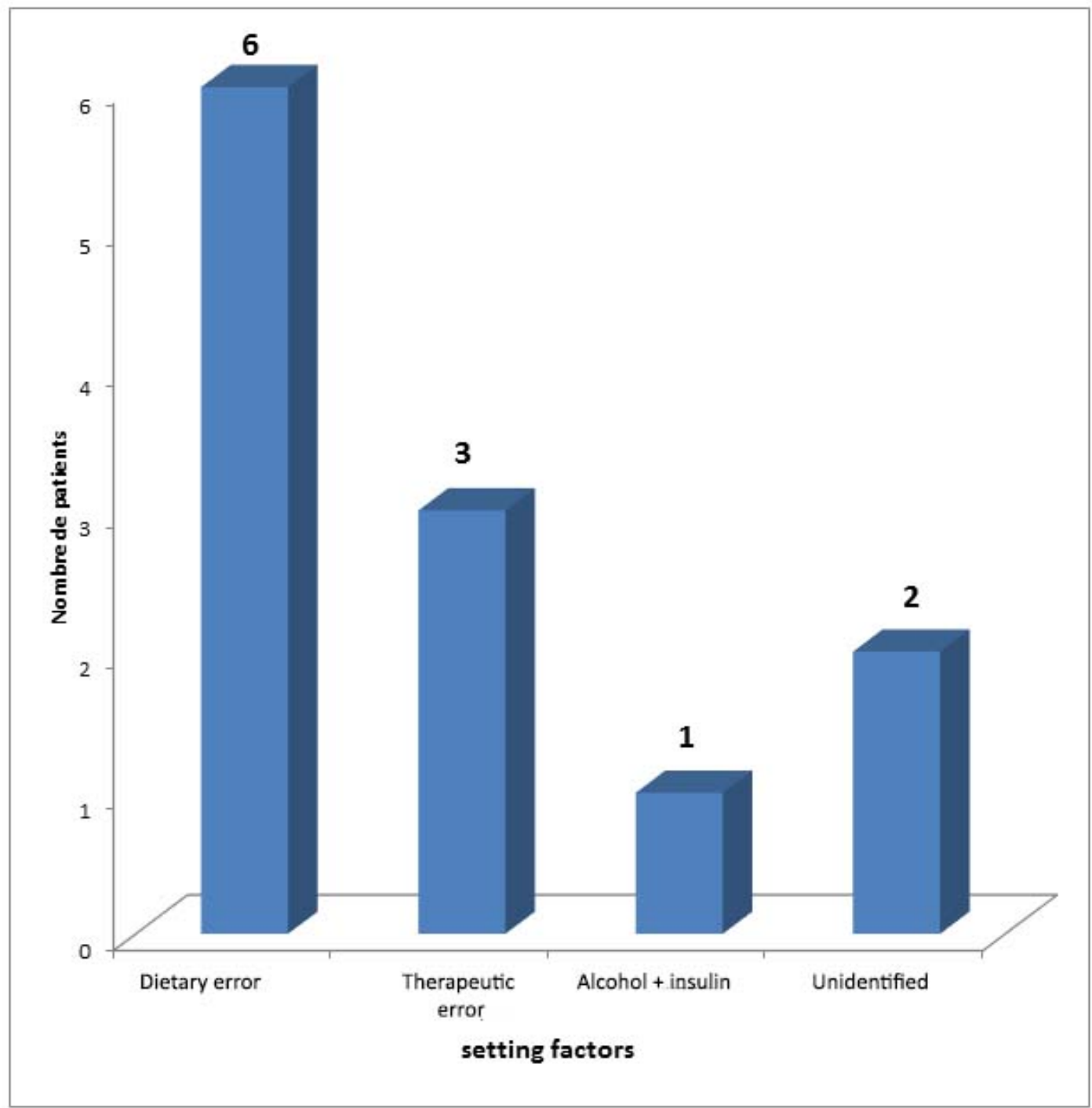

$50 \%$ (6/12) of patients had hypoglycemia by dietary error. Hypoglycemia by therapeutic error was found in $25 \%$ of patients (3/12).

Figure 3. Distribution of hypoglycemic decompensation setting factors.

years as average age of their study population and sex ratio of 1.21 in favor of females [11].

\subsubsection{Type of Diabetes}

Type2 diabetes was predominant for hyperglycemic decompensation with $60 \%(n=27)$ against $40 \%(n=18)$ for diabetes type1. The same phenomenon occurs for hypoglycemia, but in a proportion of third parties. In sum, type2 diabetes was found most (61\%) in our study population, which is conventionally known.

Mr. Ouedraogo et al. in 2000 and Sidibe, A. T., et al. in 2002 were respectively 91.77\% and 95.1\% for type2 [11] [12].

\subsubsection{Social Cover}

Our results on the phenomenon approaching that of Gninkoun [8] who found in 2009 that more than 3 out of 4 diabetics that is to say (83.52 \%) had no support or health insurance.

\subsubsection{Diabetic Land Known}

L. V. Ramanitriniaina et al. have found that subjects with diabetes was seen complicated stage represent $25 \%$ of their study population [9], the result of $23 \%$ that we found is approaching. This proportion corresponds 


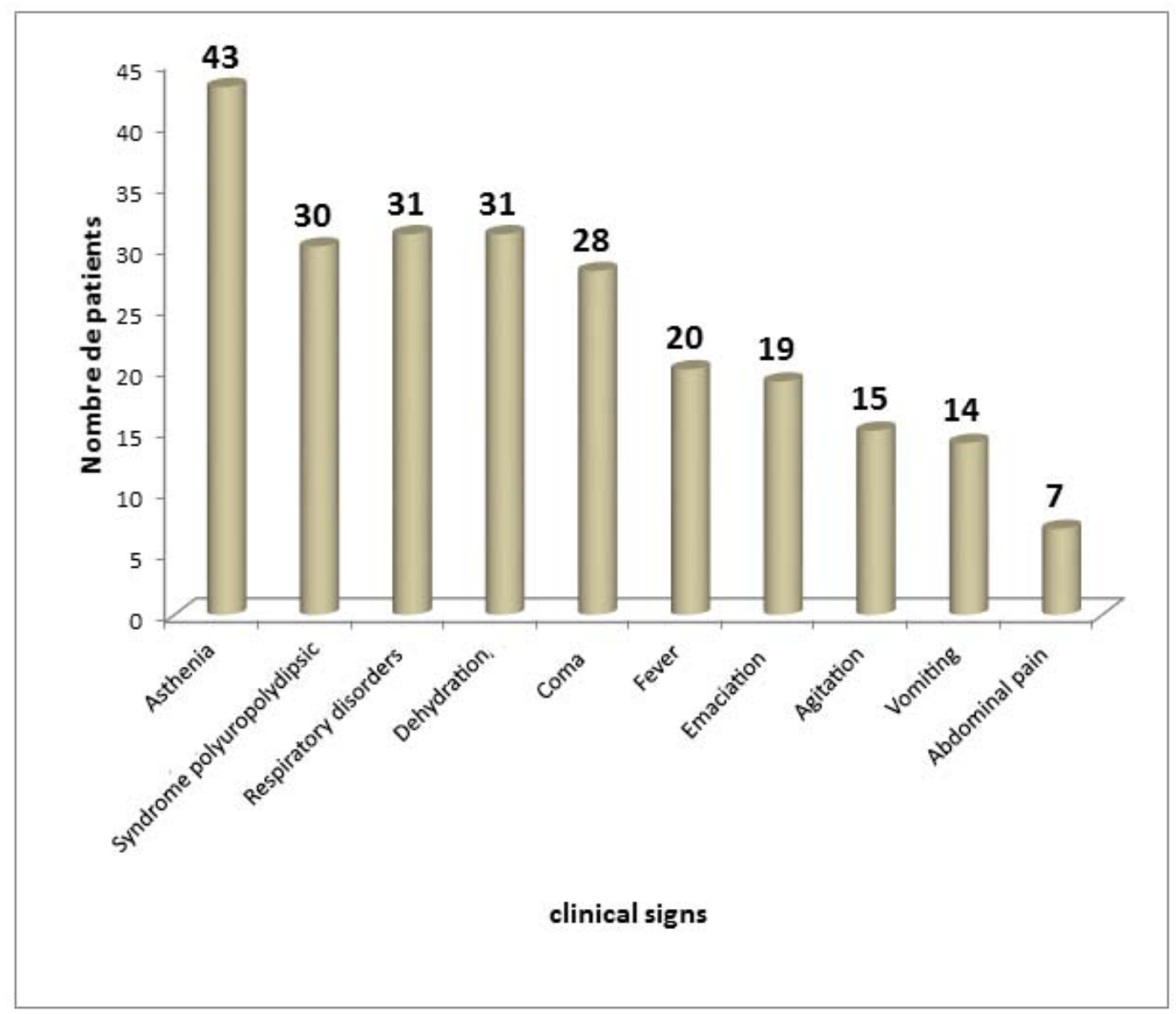

96\% (43/45) of the patients had clinical signs such as hyperglycemic decompensation asthenia. A polyuropolydipsic syndrome, respiratory problems, dehydration, coma were found in roughly the same proportions $67 \%$ (30/45).

Figure 4. Distribution of patients according to clinical characteristics of hyperglycemic decompensation.

to that reported in the literature. Morocco, diabetes is diagnosed during metabolic, cardiovascular and degenerative complications in $25 \%$ of cases [13].

On the contrary, Gninkoun and other authors found a population in which decompensation was opening their diabetes in about $50 \%$ of the workforce [5]. This improved knowledge could be explained by the effect of some information and systematic screening sessions.

\subsection{On Clinical and Biological Plan}

The signs remain classics. The fatigue, the polyuria syndrome, coma and respiratory disorders account for $95 \%$, $79 \%, 63 \%$ and $61 \%$ for all diabetic decompensation respectively.

For hyperglycemic decompensation, respiratory problems and dehydration ranked third (69\%) after asthenia (96\%) and polyuria syndrome (80\%). The same clinical signs are found in almost the same proportions for diabetic ketoacidosis. Which is consistent with the $54 \%$ of ketoacidosis our study population.

For hypoglycemia, all patients had a sweating with asthenia (91\%) and coma (67\%), which agrees well with the literature.

Sidibe, A.T., et al. also found $100 \%$ sweating all patients hypoglycemic [11].

Coma was found in $63 \%(n=36)$ of patients in our study population with a Glasgow Coma Score average $9 / 15 \pm 2$ (3 - 13).

On the biological level, the average blood glucose levels obtained in our series was $4.075 \pm 1.152 \mathrm{~g} / \mathrm{l}$ with a range of $2.340 \mathrm{~g} / \mathrm{l}$ and $6 \mathrm{~g} / \mathrm{l}$ for diabetic ketoacidosis, similar to Gninkoun results in 2009 [5]. Traditionally, blood glucose levels are below $6 \mathrm{~g} / \mathrm{l}$ higher rates are often found in Africa, as is the case in our series, probably 


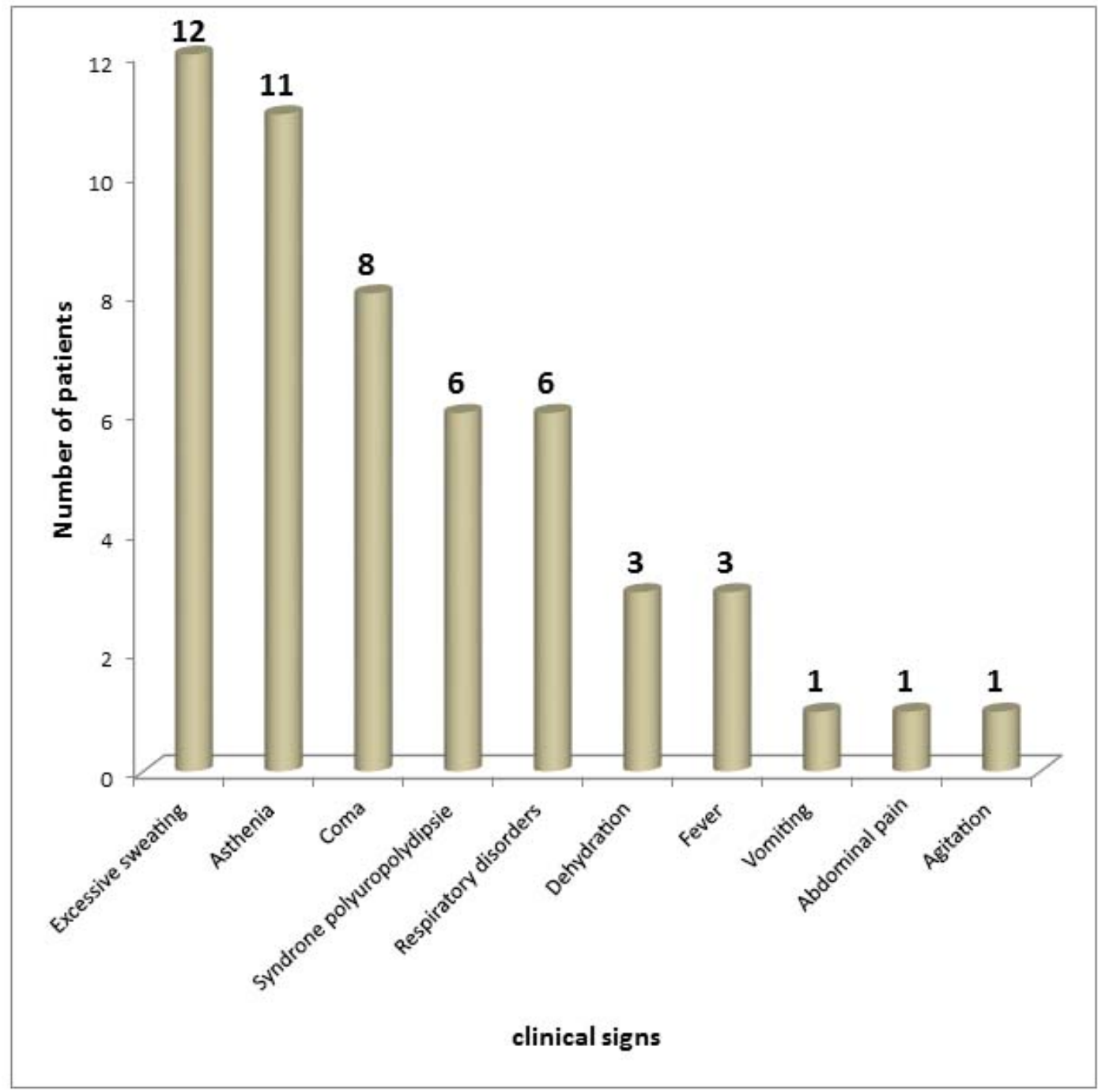

In total, $63 \%(n=36 / 57)$ of patients were hypoglycemia, be admitted in a coma or have evolved into an altered mental status. The mean GCS was $9 / 15$, with extreme $3 / 15$ to $13 / 15(\mathrm{p}=0.0000)$

Figure 5. Distribution of patients according to clinical features of hypoglycemic decompensation.

related to a late pick worsening dehydration.

All patients had a positive urine glucose $\geq 65 \%++$ et $97 \%$ had positive urine ketones $\geq 87 \%++$. These results are typically consistent with the literature and are similar to those found by Gninkoun and Wade, K.A., et al. [5] [10].

The only patient who had a negative ketonuria at the entrance was young (18 years) and had received insulin prior to admission (glucose $=4.49 \mathrm{~g} / \mathrm{l}$ and glycosuria $=4+$ ).

Serum electrolytes and additional assessment (CBC, urinalysis, blood culture, ECG, chest X-ray, etc.) remained mostly unrealized due to low purchasing power and the lack of insurance plan for our population of study.

\subsection{Setting Factors}

As Gninkoun was found in his series [5], the condition factor of decompensation most frequently found in our series is infection $(54 \%, n=30)$, cardiovascular events after $29 \%(n=15)$.

Similar outbreaks are found in the two studies are: respiratory (21\%), skin (16\%) and urine (5\%). In 1997, Mr. Ouedraogo et al. have found up to $47 \%$ lung home in their series [12]. 
The triggers of our series for hypoglycemia are dominated by dietary and therapeutic error. Mr. Ouedraogo et al. found the same thing [12]. In addition, there has been a case of alcohol consumption and fasting insulin among our patients; this raises the problem of the education of the diabetic.

\subsection{Therapeutical Plan}

\subsubsection{Waiting Time}

The average waiting time of $47 \mathrm{mn}$ found in our study is relatively long, given the delay often accused before access to the hospital, $54 \%$ of our patients had to wait 30 - 60 minutes for first aid.

\subsubsection{Measures of General Resuscitation of Patients in Coma}

Like what has been done in the work of Wade, K. A., et al. [10], all our patients in coma 63\% (n = 36) had received general resuscitative measures: implementation status and vital function monitoring, urinary catheter, nasogastric tube if necessary. Indeed, orotracheal intubation was required in $42 \%(n=15)$ of our patients had received 5 which assisted ventilation with oxygen.

Empirical antibiotic therapy (amoxicillin and clavulanic acid ouceftriaxone) was made in a 64\% (n = 23) of patients and prevention of venous thrombosis in $28 \%(n=10)$ of cases.

\subsubsection{Intensive Insulin Therapy and Fluid and Electrolyte Resuscitation}

More than 3 out of 4 patients in our series, as in the study of Gninkoun [8], have received intensive insulin therapy following different patterns: Either discontinuous all hours as an intravenous or by electric syringe pump intravenous continuous (1-10 IU) or in a pattern says to " 4 times" with subcutaneous injection every 6 h and at a fixed time. The injection scheme batch was the most used (73\%), which increases the workload of the nurse.

The use of electric syringe pump, less cumbersome and more reliable method has been carried out in $9 \%$ of cases. While she was in the systematic study Wade, A. T., et al. [10]. This low use of electric syringe pump is linked to the non-availability of these devices in functional status.

Rehydration was carried out by intake (98\%) of isotonic saline at first associated with the platform by nasogastric tube as the case water. The basic inputs were provided through the $5 \%$ glucose solution as soon as capillary blood glucose (measured hourly) was less than or equal to $2.50 \mathrm{~g} / \mathrm{l}$. Potassium and sodium are made in $86 \%$ of cases.

\subsubsection{Evolution}

The average length of stay was $\pm 4 d 5 d$ with a range of 0 to $25 d$ ( $p=0.000)$.

Of the 57 patients in our series, 63\% $(n=36)$ were kept less than 72 hours in service. Wade, K.A., et al. found as the average length of hospitalization, $\pm 7.36 \mathrm{~d} 5.05 \mathrm{~d}$ in the intensive care unit of the main hospital in Dakar [10].

As part of our study, it is under observation rather than hospitalization.

\subsection{Results Achieved}

The outcome was favorable in $56 \%(n=32)$ of cases for any failure, against $25 \%(n=14)$ deaths, $19 \%(n=11)$ of patients being transferred to the ICU for vital distress.

Wade, K. A., et al. found $17.3 \%$ of deaths in their series for diabetic ketoacidosis [10], which is very close to our result $16 \%(n=9)$.

On the contrary, Sidibe, et al. had found $14.51 \%$ of deaths for the hyperosmolar hyperglycemia [11], against 5\% in our series. But rather their series consisted of $74.2 \%$ of hyperosmolarity, against $25 \%$, the third in ours.

For Kitabchi, A. E., et al. "The mortality rate for DKA inferior to averaged 5\% with a range from 0 to over $15 \%$. These differences are explained primarily by the centres experience, patient age and the presence of comorbidity, and that of hyperosmolarity was relatively high, around 15\%" [8].

Regarding hypoglycemia, results in good agreement with what is described in the literature. Indeed, the mortality rate ranges from $0.2 \%$ to $5 \%$ irrespective of sex and diabetes type [7].

\section{Conclusions}

At the end of this work entitled "Study of the management of diabetic metabolic emergency in the national teaching hospital HKM of Cotonou”, it appears that: 
The prevalence of metabolic decompensation diabetes is $2 \%$ of the national teaching hospital HKM of Cotonou.

Average age of subjects was $49 \pm 16$ years for the hyperglycemic decompensation and $56 \pm 16$ for hypoglycemia.

The female sex was predominant with a sex ratio of 0.8 for hyperglycemia, and male predominant 3 out of 4 for hypoglycemia.

Type2 diabetes is more representative in all types of decompensation.

Infections are the prime factors of occurrence of hyperglycemic decompensation, then comes the cardiovascular accident hypertension. As for hypoglycemia, dietary and therapeutic errors were much more implicated.

The outbreaks most frequently encountered were respiratory and urinary systems.

The clinical picture is classic, dominated by fatigue, the polyuria syndrome, coma and sweating.

At least three methods of insulin have been identified in the daily practice of care. Electric syringe pump was used less.

At the level of care provided and the specific monitoring of treatment, there emerges a need for harmonization of protocols.

A lack of cooperation and exchange between the University Cuau and clinical endocrinology and metabolic diseases was noted.

The response to treatment is generally favorable. However, a death rate of $25 \%$ is cause for concern and calls for action.

\section{References}

[1] International Diabetes Federation (IDF) (2011) The Global Burden. 5th Edition, Diabetes Atlas, Brussels. http://www.idf.org/diabetesatlas

[2] Labie, D. (2007) Diabetes in Sub-Saharan Africa. Medical Sciences, 23, 320-322.

[3] Fambo, D. (2008) Prevalence of Hyperglycemia and Hypercholesterolemia in the General Population. University of Abomey Calavi, Cotonou.

[4] Djrolo, F., K. Amoussou-Guenou D., Zannou, D.H., et al. (2003) Prevalence of Diabetes Mellitus in Benin. Louvain Medical Rev. Medical Brussels.

[5] Gninkoun, J.C. (2009) Epidemiological and Clinical Profiles and Therapeutic Ketotic Decompensation of Diabetes Mellitus in the Department of Endocrinology and Metabolic Diseases of CNHU-HKM COTONOU. Thesis in MD, University of Abomey Calavi, Cotonou.

[6] Teke, N. (2003) Profile Epidemiological Clinic Acute Decompensation of Diabetes Mellitus in the General Referral Hospital of Kinshasa. Thesis in MD, University Kimbangu Simon, Kinshasa.

http://www.memoireonline.com/12/07/829/m_profil-epidemio-clinique-complications-metaboliques-aigues-diabete-su cre-kinshasa $0 . h t m l$

[7] Sobngwi, E. (2009) National Obesity Centre of Endocrinology and Diabetology, Central Yaoundé DHC Emergency Hospital in Adults. Francophone during Diabetes. World Diabetes Foundation, Cotonou.

[8] Kitabchi, A.E., Umpierrez, G.E., Murphy, B.M. and Kreisberg, R.A. (2006) Hyperglycemic Crises in Adult Patients with Diabetes. Diabetes Care, 29, 2739-2748.

[9] Ramanitriniaina, L.V., Rakotonirina, E.C.J., Rakotndravoavy, L. and Rakotomanga, J.D.M. (2009) Complicated Diabetes: Défis. Médecine Black Africa, No. 5611. 545-548.

[10] Wade, K.A., Niang, E.H.M., Soumare, C.A.T., Diallo, A., Tine, Y. and Diatta, B. (2011) Prise in Charge of Diabetic Ketoacidosis in the Medical Intensive Care Unit of the Main Dakar/SENEGAL Hospital. African Journal of Anesthesiology and Emergency Medicine (RAMUR), 16.

[11] Sidibe, A.T., Cisse, I., Dembele, M., Bocoum, A.I., Aboubacrine, S.A.G., Diarra, S., Traore, A.K. and Traore, H.A. (2005) Diabetes Emergencies: Place Hyperosmolarity. Medicine Black Africa, No. 5210, 552-554.

[12] Ouedraogo, Mr., Ouedraogo, M.S., Ouedraogo, A.S., Zoubga, A.Z., Ouedraogo, G. and Drabo, Y.J. (2001) Place Respiratory Complications in Acute Complications of Diabetes Mellitus National Hospital Yalgado Ouedraogo. Medicine Black Africa, 48, 254-256.

[13] Fattorusso, V. and Ritter, O. (2006) Clinical Vademecum: From Diagnosis to Treatment. 18th Edition, Elsevier/Masson, 1246-1265. 
Scientific Research Publishing (SCIRP) is one of the largest Open Access journal publishers. It is currently publishing more than 200 open access, online, peer-reviewed journals covering a wide range of academic disciplines. SCIRP serves the worldwide academic communities and contributes to the progress and application of science with its publication.

Other selected journals from SCIRP are listed as below. Submit your manuscript to us via either submit@scirp.org or Online Submission Portal.
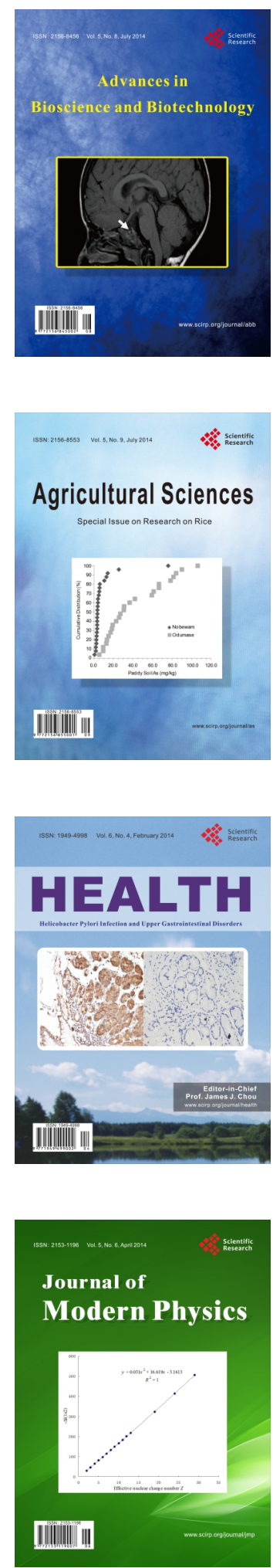
